\title{
Preoperative predictors of malignancy in lesions classified as \\ BETHESDA IV
}

Sefika Burcak Polat ${ }^{1}$, Berna Ogmen ${ }^{1}$, Husniye Baser ${ }^{1}$,Nuran Sungu², Gurkan Dumlu ${ }^{3}$, Reyhan Ersoy ${ }^{1}$, Bekir Cakir ${ }^{1}$

\begin{abstract}
1Yildirim Beyazit University, Faculty of Medicine, Department of Endocrinology and Metabolism, Ankara, Turkey 2Yildirim Beyazit University, Faculty of Medicine, Pathology Department, Ankara, Turkey 3Yildirim Beyazit University, Faculty of Medicine,General Surgery Department
\end{abstract}

\section{Introduction}

Differential diagnosis of any thyroid nodule is difficult for the clinicians if it is a follicular lesion. Follicular lesion definition includes follicular adenoma, follicular carcinoma (FC), or follicular variant papillary carcinoma (FVPTC). Fine-needle aspiration (FNA) biopsy of the thyroid is the best diagnostic procedure for the preliminary assessment of thyroid nodules. FNA biopsy of the thyroid is a very effective screening test and has resulted in a decreased incidence of unnecessary surgery . FNA biopsy can effectively diagnose benign thyroid conditions and the majority of cases of papillary thyroid carcinoma (PTC), which is the most common malignant neoplasm of the thyroid. However because of the cytolgical similarities among different subtypes of follicular lesions the ability of the FNA decreases and being subjective depending on the observer. In addition to that differentiation of malignant follicular lesions from benign ones requires demonstration of the capsular/vascular invasion which can only be determined after surgical resection and histologic examination of the entire capsule . In this study, we aimed to investigate the the clinical, cytological, and sonographic profile of patients who had a confirmed diagnosis of a follicular thyroid neoplasm on histology in an attempt to identify distinguishing features a benign or malignant follicular thyroid neoplasm

\section{Methods}

Eighty consecutive patients with an operated follicular thyroid neoplasm at a tertiary hospital from 2007 to 2014 were reviewed. Age, gender, symptoms, history, physical findings, nodule size, sonographic, cytologic, and final pathologic results were recorded. Malignant and benign groups were compared according to preoperative clinical and imaging features.

\section{Results:}

34 of 102 nodules were malignant where as 68 were benign. Gender distribution, baseline thyroid function tests and thyroid autoantibody positivity were similar between the benign and malignant groups. Family history of differentiated thyroid cancer (DTC) was significantly higher in the malignant group ( $\mathrm{p}=0.002)$. Regarding to ultrasonographic parameters, nodule volume and vascularity were significantly greater in the malignant nodules ( $\mathrm{p}=0.04$ and 0.008 , respectively) Presence of microcalcification / irregular macrocalcification was also higher in the malignant group compared to benign group $(\mathrm{p}=0.017)$. When we subdivided malignant nodules as FVPTC (18 lesions) and FC (16 lesions), microcalfication was significantly more common in FVPTC $(\mathrm{p}=0.022)$.

\section{$>$ Conclusion:}

Our study demonstrates that patients with benign and malignant follicular thyroid lesions are different with respect to several presenting clinical features. Family history and certain ultrasonographic parameters might be helpful in preoperative differentiation of benign and malignant follicular neoplasms. A combination ofthose with both FNA and molecular results may help us to decide management of patients with follicular thyroid lesion 